\title{
Synergy between rhBMP-2 and IKK-Inhibitor PS-1145 Delivered via a Porous Biodegradable Polymer Implant
}

David Carr ${ }^{1,2}$, Nicole Y.C Yu ${ }^{1,2}$, Jane Fitzpatrick ${ }^{4}$, Lauren Peacock ${ }^{1}$, Kathy Mikulec ${ }^{1}$, Andrew J. Ruys ${ }^{2}$, Justin C. Cooper-White ${ }^{4}$, David G. Little $^{1,3}$ and Aaron Schindeler ${ }^{1,3 *}$

${ }^{1}$ Department of Orthopaedic Research \& Biotechnology, The Children's Hospital at Westmead, Locked Bag 4001, Westmead, NSW 2145, Australia

${ }^{2}$ School of Aerospace, Mechanical and Mechatronic Engineering, J07 University of Sydney, NSW 2006, Australia

${ }^{3}$ Discipline of Paediatrics and Child Health, Faculty of Medicine, A27 University of Sydney, NSW 2006, Australia

${ }^{4}$ Tissue Engineering and Microfluidics Laboratory, Australian Institute for Nanotechnology and Bioengineering, University of Queensland, Brisbane, QLD, Australia

\begin{abstract}
Critical-sized bone defects, whether caused by congenital malformation, tumor resection, trauma, or implant loosening, remain a major challenge for orthopaedic management. In this study we describe a bone tissue engineering approach in mice for the co-delivery of recombinant human Bone Morphogenetic Protein-2 (rhBMP-2) and the IKK inhibitor PS-1145.

Scaffold implants were manufactured from poly(lactide-co-glycolide)(PLGA) by Thermally-Induced Phase Separation (TIPS), with rhBMP-2 $(10 \mu \mathrm{g})$ and the IKK inhibitor PS-1145 $(0 \mu \mathrm{g}, 40 \mu \mathrm{g}$ or $80 \mu \mathrm{g})$ incorporated into the polymer. These scaffolds were then surgically implanted into the hind limb muscle of C57BL6/J mice. One group of mice also received systemic $50 \mathrm{mg} / \mathrm{kg}$ PS-1145 (days 11-20). Specimens were harvested at week 3 for X-ray and microCT analyses and descriptive histology.

Local and systemic delivery PS-1145 both significantly increased the net rhBMP-2 induced bone at 3 weeks. A maximal response was seen with the $40 \mu \mathrm{g}$ PS-1145 group, although there was no significant difference between the $40 \mu \mathrm{g}$ and $80 \mu \mathrm{g}$ PS-1145 regimens. No local cytotoxicity was seen with either dose of PS-1145. In summary, local co-delivery of rhBMP-2 and PS-1145 via a porous PLGA scaffold represents a new tissue engineering approach for maintaining new bone in an unloaded environment.
\end{abstract}

Keywords: PS1145; IKK inhibitor; rhBMP-2; Bone morphogenetic protein; Bone tissue engineering; Biodegradable polymer scaffold; Thermally induced phase separation

\section{Introduction}

Treatment of critical-sized bone defects remains a challenge for orthopaedic medicine. Such defects require exogenous osteogenic stimulation in order for them to heal [1], autograft being the current gold standard. Autograft is not without its limitations, including its availability and complications associated with donor site morbidity [2-8]. Other alternatives, such as allograft or bone transport using the Ilizarov technique, have utility but key limitations. Allograft has a reduced osteoinductive capacity and has an intrinsic disease and rejection risk $[4,6,8]$, while bone transport can be prolonged and uncomfortable [9-11].

Bone tissue engineering is a developing field of research that shows many promising possibilities for the treatment of critical size bone defects [6]. Giannoudis et al.[12] have described four essential components required to facilitate engineered bone as being (1) an osteoconductive matrix scaffold; (2) osteoinductive growth factors; (3) a cell population with osteogenic capacity; and (4) a mechanically stable environment. In this study, we aimed to create a biocompatible porous scaffold for the delivery of osteoinductive growth factors. In mice, new bone was induced within the muscle compartment; this tissue contains cells with osteogenic potential [13], but represents a mechanically unloaded environment. To compensate for the biomechanical insufficiency, we have trialed local delivery of an IKK inhibitor to prevent osteoclast-mediated bone loss.

A range of materials have been proposed for scaffolds including metals, ceramics and polymers, both natural and synthetic. Synthetic polymers have become the most commonly used material for bone tissue engineering, particularly the poly( $\alpha$-hydroxyacids), which have great chemical versatility $[8,14-16]$. Numerous methods have been used to fabricate poly( $\alpha$-hydroxyacids) scaffolds to optimize the essential properties of a scaffold (porosity, pore size, surface properties, osteoinductivity, mechanical properties, and biodegradability) Solvent-casting and particulate leaching [17], emulsion freeze-drying [18], electrospinning [19], gas foaming [20], the various types of rapid prototyping [21] and most recently thermally induced phase separation (TIPS) [22] have all been described. We have utilized a TIPS-based method that allows for rapid fabrication of high porosity scaffolds with defined pore sizes. Poly(lactide-co-glycolide) (PLGA) was used as a copolymer of poly(lactic acid) (PLA) and poly(glycolic acid) (PGA) to generate a material capable of degrading into non-toxic, natural metabolites [14].

Osteoinductive growth factors are an integral component of bone tissue engineering. Recombinant human BMP-2 and BMP-7 are potent anabolic agents that are reported to have an efficacy comparable to autograft and act to potently stimulate the differentiation of

*Corresponding author: Aaron Schindeler, Department of Orthopaedic Research \& Biotechnology, The Children's Hospital at Westmead, Locked Bag 4001 Westmead, NSW 2145, Australia, Tel: +61-2-98451451; Fax: +61-2-98453078; E-mail: aaron.schindeler@sydney.edu.au

Received April 03, 2012; Accepted April 24, 2012; Published April 26, 2012

Citation: Carr D, Yu NYC, Fitzpatrick J, Peacock L, Mikulec K, et al. (2011) Synergy between rhBMP-2 and IKK-Inhibitor PS-1145 Delivered via a Porous Biodegradable Polymer Implant. J Tissue Sci Eng S1:003. doi:10.4172/2157-7552. S1-003

Copyright: (c) 2011 Carr D, et al. This is an open-access article distributed under the terms of the Creative Commons Attribution License, which permits unrestricted use, distribution, and reproduction in any medium, provided the original author and source are credited. 
osteoprogenitors [23]. Recombinant BMPs are reported to lessen times to union in fractures [24-26], and can facilitate complete healing in non-union fracture defects otherwise unable to heal without intervention [27]. However, BMPs have shown potential to promote osteoclast formation [28] and excessive bone resorption [29].

Unloaded bone is progressively resorbed, as per Wolff's law [30], both in skeletal remodeling and bone callus remodeling. In the context of critical-sized defects, premature bone catabolism can diminish the rate at which strength can be restored, thus delaying the time to union [31]. Similarly, for tissue engineering implants, the site of regeneration is often poorly loaded. To overcome this intrinsic deficiency, anticatabolic drugs such as bisphosphonates have been proposed as adjunctive agents [32]. Local bisphosphonate dosing in a solvent-cast PDLLA implant was found to augment the net bone remaining at the implant site at 3 weeks in a mouse [33] but only within a narrow dose window. At high local doses it was found to produce toxic effects that decreased bone formation, and this was comparable to a prior bone defect study [34]. It was thus hypothesized that alternative anti-catabolic agents may have a broader effective dose range than bisphosphonates when delivered locally.

IKK-inhibitors are emerging as potent anti-resorptives, but they have not been explored in the context of bone tissue engineering. IKKinhibitors target the nuclear factor- $\mathrm{kB}$ (NF- $\mathrm{kB}$ ) signaling pathway, which has a pivotal role in the regulation osteoclastogenesis, as well as the general inflammatory response [35]. IKK consists of two catalytic subunits, IKKa and IKK $\beta$, and a regulatory subunit IKK $\gamma$ or NEMO. IKK phosphorylates inhibitor of NF- $\mathrm{kB}$ ( IkB) leading to its degradation and allowing the translocation of NF- $\mathrm{KB}$ from cytoplasm to nucleus where it can induce expression of target genes, which regulate the differentiation of osteoclast precursors into mature multinucleated active osteoclasts [35-37]. IKK-inhibitors can induce apoptosis in osteoclast precursors and inhibit osteoclastogenesis and bone erosion [38-40], which make them potential therapeutics for osteoporosis and rheumatoid arthritis. Dai et al.[41] developed a NEMO-Binding Domain peptide (NBD) that in vitro inhibited the differentiation of osteoclasts whilst also inducing apoptosis and in vivo attenuated osteoclastogenesis and inflammation-induced bone loss. IKK inhibition increased trabecular bone mass and Bone Mineral Density (BMD) while preventing osteoporotic bone loss in ovariectomized mice [36]. PS-1145 is an IKK-inhibitor that has shown promise for the treatment of cancer-induced lytic bone disease through blocking osteoclast maturation [42]. Pharmacological inhibitors of IKK, such as PS-1145, offer a novel approach to the suppression of early catabolism in the healing of large bony defects through inhibition of pathways necessary for osteoclast survival, differentiation and function.

In this study, we aimed to investigate the potential of the IKK inhibitor PS-1145 as an anti-catabolic drug to be co-delivered with rhBMP-2 in a bone tissue engineering system. We also wanted to determine the relative effectiveness of local versus systemic dosing of PS-1145. Using a TIPS-produced porous PLGA scaffold, we hypothesized that the PS-1145 would show synergy with rhBMP-2 and result in greater volumes of unresorbed bone, as a result of its inhibitory action on osteoclasts.

\section{Materials and Methodology}

\section{Pharmaceuticals}

INFUSE $^{\mathrm{TM}}$ rhBMP-2 was purchased from Medtronic Australasia and supplied as a lyophilized protein. PS-1145 (>98\% HPLC) was purchased from Sigma Aldrich Australia. Systemically-delivered dosages of PS-1145 were mixed in 10\% dimethylsulfoxide (DMSO, Sigma Aldrich) and 90\% sterile water.

\section{Polymer carrier materials and manufacturing}

The thermally induced phase separation (TIPS) technique was utilized to fabricate 40 PLGA scaffolds of $90 \%$ porosity. The methods used were based on those of Cao et al. [43] and Jack et al. [44]. The copolymer PLGA (LactelDurect. 50:50 Poly(D,L)-lactide-co-glycolide) was added to 1,4-dioxane solvent (LABSCAN) at a concentration of $125 \mathrm{mg} / \mathrm{ml}$. INFUSE ${ }^{\mathrm{TM}}$ rhBMP-2 (Medtronic) and PS-1145 powder were added to 1,4-dioxane and mixed to the PLGA/dioxane solution as required to incorporate $10 \mu \mathrm{g}$ rhBMP-2 and $40 \mu \mathrm{g}$ or $80 \mu \mathrm{g}$ PS- 1145 per $1 \mathrm{~mm}$ disc.

Drug/PLGA/dioxane solutions were vortexed and pipetted into $3 \mathrm{~mm}$ long glass tubes that were sealed and slow-quenched in a temperature controlled water bath (Polyscience Model 9112) $\left(\mathrm{T}=25^{\circ} \mathrm{C}\right.$ cooled at a rate of $-0.5^{\circ} \mathrm{C} / \mathrm{min}$ to $-10^{\circ} \mathrm{C}$ ). This induced porosity via a solid-liquid phase separation. Glass tubes were then submerged in liquid nitrogen $\left(-80^{\circ} \mathrm{C}\right)$ and carefully removed from the polymer with use of a hammer. The solidified PLGA scaffold was vacuum-dried at $<1 \times 10^{-3}$ mbar to remove residual dioxane for 4 hours. The cylindrical scaffolds were cut into $1 \mathrm{~mm}$ thick pellets for implantation and stored at room temperature under vacuum.

\section{Animal care and surgery}

Female C57BL6/J wild type mice (8-10 week, average weight 18 g) were purchased from the Animal Resources Centre and allowed to acclimatize for 7 days prior to surgery. Mice were housed in autoclavable polypropylene solid boxes with stainless steel lids and polycarbonate water bottles with food and mouse chow supplied ad libitum. Ethics approval was obtained for all procedures from the local Animal Ethics Committee (Approval K294).

Ectopic bone formation was induced in a quadriceps muscle pouch following surgical implantation of rhBMP-2-loaded PLGA carrier. The surgical model was based on previously published methods where rhBMP-2 was delivered in a carboxy-methylcellulose carrier [45]. In brief, anesthesia was induced and maintained using inhaled isofluorane and an intraperitoneal (i.p.) injection of $35 \mathrm{mg} / \mathrm{kg}$ ketamine and 4.5 $\mathrm{mg} / \mathrm{kg}$ xylazine. The operative site was trimmed and prepared with povidone-iodine. A muscle pouch was created using a scalpel in the right hind limb, in line with the femur. The polymer pellet was inserted and the muscle and skin closed using a 5-0 Vicryl suture (Ethicon Inc). Following surgery, animals were recovered on a heated pad and placed in recovery cages. Post-operative pain was managed with subcutaneously injected buprenorphine $(0.05 \mathrm{mg} / \mathrm{kg})$ and dehydration was managed by saline injection, as required. Animals were monitored by weekly weighing.

\section{Experimental design}

The effects of 2 different local dosages and 1 systemic dosage of PS-1145 on rhBMP-2 induced bone formation were compared. Mice were assigned to each group: rhBMP-2 only controls $(n=8)$; and rhBMP-2 with $40 \mu \mathrm{g}(\mathrm{n}=8)$ or $80 \mu \mathrm{g}(\mathrm{n}=8)$ local PS-1145; and rhBMP-2 with $50 \mathrm{mg} / \mathrm{kg}$ systemic PS-1145 $(\mathrm{n}=8)$ as summarized in (Table 1 ). Mice receiving systemic PS-1145 were dosed i.p. from day 11 to day 20. Animals were euthanized at 3 weeks post-operatively. Whole hind limbs were harvested post-mortem, fixed in $4 \%$ paraformaldehyde, and stored in $70 \%$ alcohol for radiographic and histological analysis. 


\section{Radiographic analysis}

The placement of the ectopic bone nodules relative to the femur was determined by radiography using a digital X-ray machine (Faxitron X-ray Corp). Samples where ectopic bone had fused with the femur or had migrated post-operatively out of the muscle pouch were excluded as per (Table 1).

Specimens were examined by micro-computed tomography (microCT) scanning using a SkyScan 1174 compact microCT scanner (SkyScan). The primary outcome measure for the study was total bone volume of the entire ectopic bone nodule. Samples were scanned in $70 \%$ ethanol at $8.7 \mu \mathrm{m}$ pixel resolution; $0.5 \mathrm{~mm}$ aluminium filter, $50 \mathrm{kV}$ X-ray tube voltage and $800 \mu \mathrm{A}$ tube electric current. The images were reconstructed using NRecon, version 1.5.1.5 (SkyScan). Bone volume $\left(\mathrm{BV}, \mathrm{mm}^{3}\right)$ of the entire ectopic bone nodule was analyzed with CTAnalyser software, version 1.10.1.0 (SkyScan). A global threshold representing bone (60-255) was defined following analysis of consecutive two-dimensional slices of bone samples on CT Analyser software. Representative three-dimensional bone pellets were reconstructed with transaxial slices (100 slices) from the pellet's midsection, using CTVol Realistic Visualization software version 2.1.0.0 (SkyScan). Representative ectopic bone samples from each group were selected as having a BV closest to the group's mean. Defining the Region of Interest (ROI) for analysis allowed the comparison of internal trabecular bone with the cortical bone forming a sheath around the exterior of the nodule. Phantom materials of known mineral density were microCT scanned under the same conditions as samples and used to calibrate CT Analyser to calculate average bone mineral density for each sample.

\section{Histological analysis}

For paraffin histology, representative ectopic bone nodule samples were decalcified and embedded in paraffin blocks. $5 \mu \mathrm{m}$-thick sections were cut using a Leica RM 2155 Microtome (Leica Microsystems). Sections were stained with Hematoxylin \& Eosin (H \& E), Alcian blue \& Picrosirius red for bone and cartilage, and Tartrate Resistant Acid Phosphatase (TRAP) for osteoclasts. Stained sections were scanned with ScanScope digital slide scanner (Aperio Technologies), and images were captured with ImageScope software (Aperio Technologies).

\section{Statistical analysis}

For data analysis, group sizes $<10$ prohibited tests of normality, necessitating non-parametric statistical tests for significance. Kruskal Wallis and Mann Whitney U tests were performed using SPSS Statistics version 17 (SPSS Inc). Statistical significance was set at $\alpha<0.05$.

\section{Results}

Pellets containing rhBMP-2 only (Groups 1 and 4 ) or rhBMP-2 and PS-1145 (Groups 2 and 3) were surgically implanted into C57B6/J mice. One mouse died during recovery and six mice were excluded due to pellet misplacement (operatively) or migration (post-operatively). Specimens were harvested after 3 weeks and all samples underwent $\mathrm{X}$-ray and microCT scanning followed by histological processing. The primary outcome measure for the study was total bone volume (BV, $\mathrm{mm}^{3}$ ) of the entire ectopic bone nodule, as quantified by microCT. Descriptive histology of the effects of PS-1145 on bone was a secondary outcome.

\section{Bone nodule X-ray and MicroCT analyses}

X-rays indicated that both systemic and local PS-1145 treatment led to larger, more radiopaque bone nodules after 2 and 3 weeks. MicroCT scans of each ectopic bone nodule were performed, and the bone volume $\left(\mathrm{mm}^{3}\right)$ in each sample was quantified (Figure 1). All PS-1145 treatment groups showed significant increases in net bone formation relative to the rhBMP-2 alone control. The $40 \mu \mathrm{g}$ local PS1145 dose showed the most prominent increase in bone volume from control ( $111 \%$ increase, $\mathrm{p}<0.02)$. Both the $80 \mu \mathrm{g}$ local PS- 1145 dose and $50 \mathrm{mg} / \mathrm{kg}$ daily PS- 1145 were associated with increases in bone volume ( $87 \%$ increase, $\mathrm{p}<0.03$ for local and $55 \%$ increase, $\mathrm{p}<0.05$ for systemic). Intra-group comparison of the different PS-1145 amounts and dosing regimens indicated none were significantly different from each other $(\mathrm{p}>0.1)$.

Qualitatively, it was seen that bone nodule samples showed the formation of a pseudo-cortex around a trabecular interior. The PS-1145 local delivery groups (2 and 3) showed greater trabecular retention by X-ray (Figure 1B) and more clearly by microCT transaxial cross-section reconstructions (Figure 1C). The interior bone was quantified using CTAn software and found to be significantly greater in all PS-1145-treated groups with 202\%, 213\%, and 201\% increases in internal trabecular-like bone formation relative to control in the 40 $\mu \mathrm{g}$ local, $80 \mu \mathrm{g}$ local, and $50 \mathrm{mg} / \mathrm{kg}$ systemic dose groups, respectively (all $\mathrm{p}<0.05$ ) (Figure 2A). Trabecular BV/TV was calculated by dividing the trabecular bone volume by the total internal volume of the nodule, and increases of $99 \%, 93 \%$, and $115 \%$ were recorded in the $40 \mu \mathrm{g}$ local, $80 \mu \mathrm{g}$ local and $50 \mathrm{mg} / \mathrm{kg}$ systemic dose groups respectively, compared to control $(\mathrm{p}<0.05)$ (Figure $2 \mathrm{~B})$. The low trabecular BV/TV found in the study was attributed to the ectopic bone nodule being mechanically unloaded with resorptive pseudo-marrow space. The quantity of bone in the pseudo-cortex growth showed significant increases in the $40 \mu \mathrm{g}$ and $80 \mu$ g local PS- 1145 groups ( $93 \%$ and $63 \%$, respectively, $\mathrm{p}<0.05$ ). The systemic PS- 1145 group had $26 \%$ increase cortical volume that was not significant $(\mathrm{p}=0.2$ ) (Figure $2 \mathrm{C}$ ). Bone mineral density (BMD) was calculated using CTAn software and as expected the PS-1145 treated samples showed significant increases. The $40 \mu \mathrm{g}$ local, $80 \mu \mathrm{g}$ local and systemic PS-1145 groups had increases of $64 \%(\mathrm{p}=0.046), 39 \%(\mathrm{p}=0.1)$ and $68 \%(\mathrm{p}=0.03)$ respectively, relative to controls (Figure $2 \mathrm{D})$.

\section{Bone nodule histology}

Prior staining of histological sections of solvent cast PDLLA containing rhBMP-2 showed poor biodegradation of the scaffold, even after 8 weeks [33]. In contrast, staining with Alcian Blue/Picrosirius Red $(\mathrm{AB} / \mathrm{PR})$ for cartilage and mineralized tissues showed comparatively greater degradation of the porous PGLA scaffold by 3 weeks (Figure 3A-D).

Each sample consisted of a bone sheath around an interior

\begin{tabular}{|l|l|l|l|l|}
\hline Group & Scaffold & Osteoinductive agent & Anti-resorptive agent & Mice \\
\hline 1 & Porous PLGA & rhBMP-2 $(10 \mu \mathrm{g})$ & None & $\mathrm{N}=8$ \\
\hline 2 & Porous PLGA & rhBMP-2 $(10 \mu \mathrm{g})$ & $\mathrm{PS}-1145($ low $40 \mu \mathrm{g})$ & $\mathrm{N}=8$ \\
\hline 3 & Porous PLGA & rhBMP-2 $(10 \mu \mathrm{g})$ & $\mathrm{PS}-1145$ (high $80 \mu \mathrm{g})$ & $\mathrm{N}=8$ \\
\hline 4 & Porous PLGA & rhBMP-2 $(10 \mu \mathrm{g})$ & $\mathrm{PS}-1145($ systemic $50 \mathrm{mg} / \mathrm{kg})$ & 3 \\
\hline
\end{tabular}

Table 1: Study Details. 
Citation: Carr D, Yu NYC, Fitzpatrick J, Peacock L, Mikulec K, et al. (2011) Synergy between rhBMP-2 and IKK-Inhibitor PS-1145 Delivered via a Porous Biodegradable Polymer Implant. J Tissue Sci Eng S1:003. doi:10.4172/2157-7552.S1-003

containing islands of bone as well as a pseudo-marrow space containing adipocytes, white blood cells and red blood cells. Adipocytes were readily identifiable by cell morphology and were most abundant towards the interior of the nodule in all sample groups.

$\mathrm{AB} / \mathrm{PR}$ staining showed that the control group had very little trabecular bone islands present within the nodule at the 3 week time point (Figure3A). There was a relatively thin cortical-like bony sheath surrounding the nodule $(\mathrm{Co})$, with some trabecular structures formed nearby the sheath (T). In contrast, the local $40 \mu \mathrm{g}$ and $80 \mu \mathrm{g}$ PS- 1145 local dose groups showed large volumes of retained bone formation (Figure $3 \mathrm{~B}$ and $3 \mathrm{C}$ ), the sheath was thicker and discontinuous in appearance compared to the control group and had attached trabeculae that protruded into the bone marrow. A number of bony islands formed throughout the nodule (B), which were smoothly contoured trabecular bone structures of multiple osteocytes resting within their lacunae and surrounded by mineralized tissue. A third unusual structure of mineralized bone tissue was also witnessed - clusters of disorganized presumably woven bone that stained a dark red on the $A B / P R$ stain. The $50 \mathrm{mg} / \mathrm{kg}$ PS-1145 systemic dose group had similar features to the local dose groups, but had a much smaller volume of retained bone structures within the nodule and little if any woven bone.

TRAP staining was performed to detect pre-osteoclasts and osteoclasts (Figure 3F-I) and TRAP+ cells were abundant in the control group (Figure $3 \mathrm{~F}$ ), even in central regions with no bone formation. Both the $40 \mu \mathrm{g}$ and $80 \mu \mathrm{g}$ PS-1145 local dose groups displayed similar amounts of TRAP staining around the cortical sheath, but relatively low numbers of TRAP+ cells were seen in the interior trabecular bone (Figure3G). Curiously, staining was particularly intense in discrete regions (Figure $3 \mathrm{G}$ and $3 \mathrm{H}$ ). In the systemically dosed group, a similar phenomenon was observed. TRAP+ staining in pellets was found to be highly heterogeneous within each sample.

\section{Discussion}

In this study, we used a TIPS-produced porous PLGA scaffold to generate new bone in a mouse model. Bone was induced with rhBMP-2

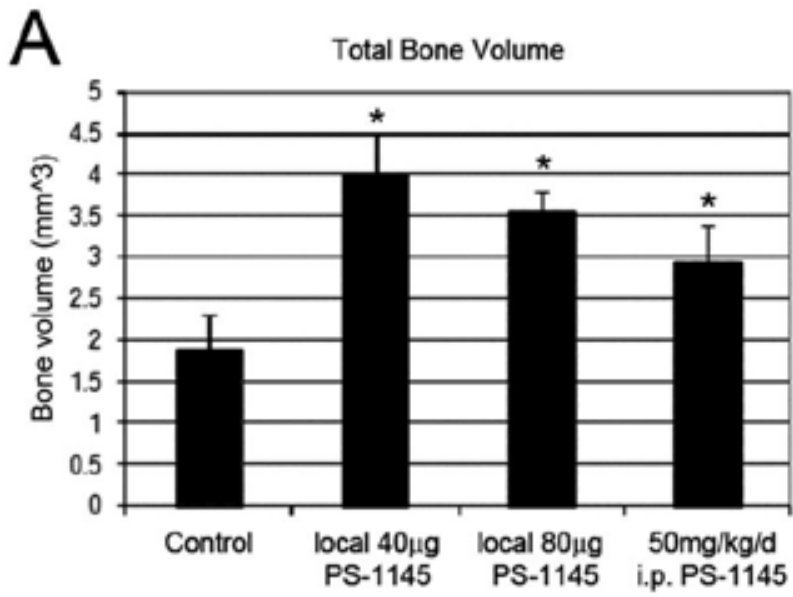

Figure 1: (A) Average total bone volume in each group, quantified by microCT. transaxial sections of representative samples. and we trialed the effects of suppressing bone resorption using an IKK inhibitor. Two local doses of PS-1145 (40 $\mu \mathrm{g}$ and $80 \mu \mathrm{g})$ and $50 \mathrm{mg} / \mathrm{kg}$ systemic PS- 1145 was compared. The $40 \mu \mathrm{g}$ locally dosed group had the greatest positive influence on net bone formation producing a $111 \%$ increase in total bone volume, followed by increases of $87 \%$ in the $80 \mu \mathrm{g}$ locally dosed group and $55 \%$ in the $50 \mathrm{mg} / \mathrm{kg}$ systemically dosed group.

We have previously investigated the use of local bisphosphonates in augmenting rhBMP-2 induced bone. These experiments showed that local Pamidronate could be as effective as systemic Pamidronate in preventing implant resorption, however the results also indicated a limited dosing window for affectivity [33]. The effectiveness of antiresorptives in maximizing bone in this model is consistent with high resorptive pressures on unloaded bone, consistent with Wolff's law [30]. However, the negative effects associated with high bisphosphonate doses [34] have the potential to be problematic in terms of clinical translation. Consequently, this study focused on the IKK inhibitor PS1145 as an alternative anti-catabolic.

The recruitment and differentiation of osteoclast precursors has been reported to be increased by mechanical unloading [30], as well as directly and indirectly augmented by rhBMP-2 $[46,47]$. Indirect methods of osteoclast stimulation are likely to be important, as rhBMP-2 induced osteoblastogenesis can result in increased osteoblast-osteoclast signaling via RANKL. By preventing the binding of the IKK subunits, PS-1145 is able to block the RANKL pathway and thus inhibit the complete differentiation of osteoclasts into mature multinucleated bone-resorbing cells. This has been demonstrated using IKK inhibitors in vitro [42] and in a mouse ovariectomy model in vivo [38]. IKK inhibition by sequestration of NEMO has been used to arrest osteoclastogenesis induced by polymethyl methacrylate wear particles [48], and the IKK inhibitor Parthenolide can inhibit osteoclastogenesis, as well as trigger apoptosis of osteoclasts at higher doses [49]. Moreover, transgenic mice with bone-specific expression of a dominant negative IKK mutant protein showed enhanced trabecular bone formation [36].

Tartrate Resistant Acid Phosphatase (TRAP) is a marker of active mature multinucleated osteoclasts [50], however it can also be

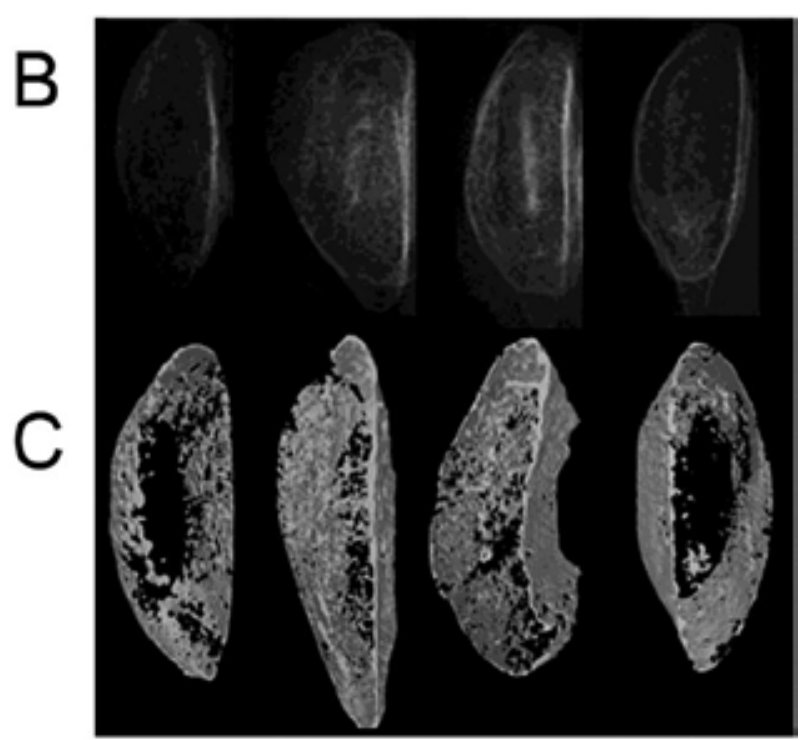

(B) X-rays of representative bone nodules. (C) microCT reconstruction of 100 
Citation: Carr D, Yu NYC, Fitzpatrick J, Peacock L, Mikulec K, et al. (2011) Synergy between rhBMP-2 and IKK-Inhibitor PS-1145 Delivered via a Porous Biodegradable Polymer Implant. J Tissue Sci Eng S1:003. doi:10.4172/2157-7552.S1-003

A

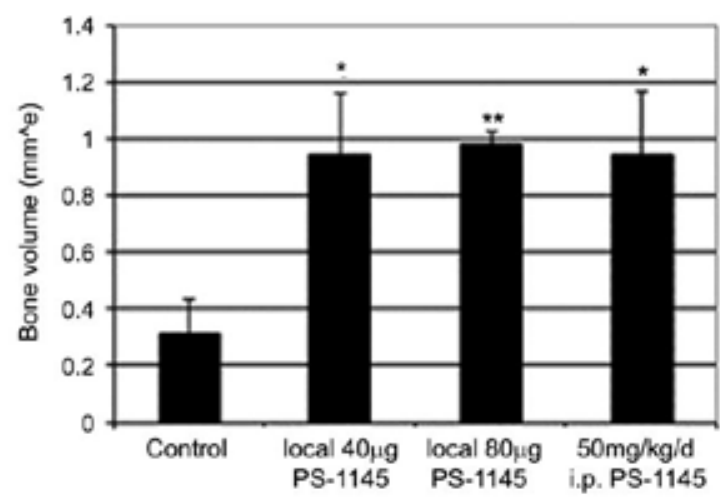

0

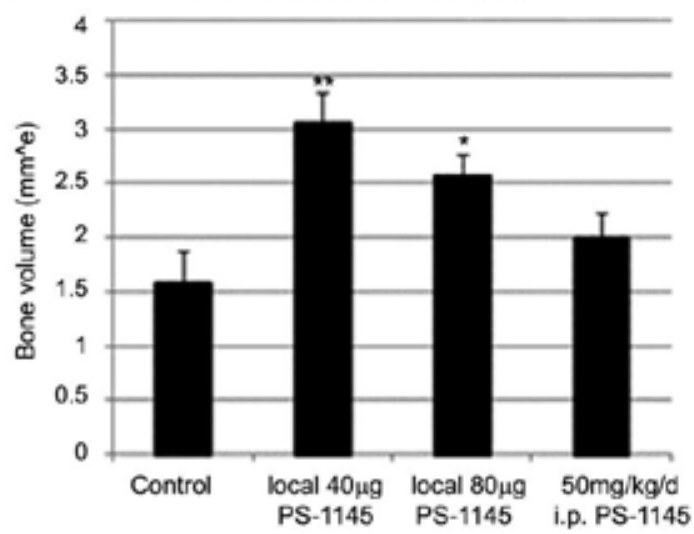

B

Trabecular BV/TV (\%)
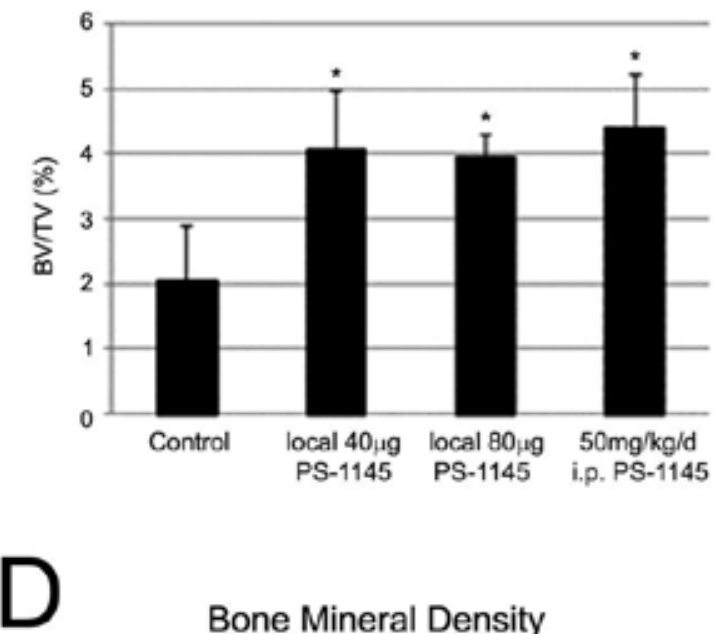

Bone Mineral Density

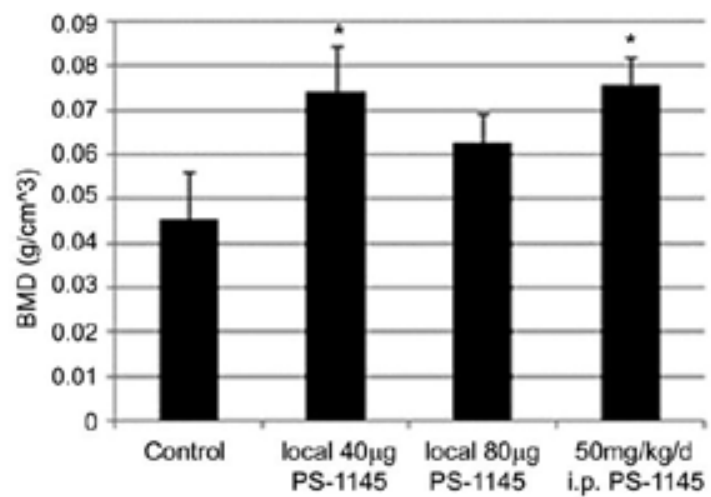

Figure 2: Values obtained from MicroCT analysis. (A) Trabecular Bone Volume, (B) Trabecular BV/TV, (C) Volume of the pseudo-cortex, and (D) Bone Mineral Density. Significance is calculated relative to the rhBMP-2 only control group $\left({ }^{*} p<0.05,{ }^{* *} p<0.01\right)$.

expressed by inactive multinucleated or immature mononuclear preosteoclasts. Local treatment with PS-1145 was descriptively observed to decrease TRAP staining in the interior trabecular bone but not to significantly influence staining in the cortical sheath. Local PS-1145 pellets showed increased bone volume by microCT, consistent with decreased overall resorption. Despite the observable effect of local PS1145 on interior, but not peripheral TRAP + cell numbers, both cortical bone volume and trabecular BV/TV were approximately 2 -fold greater in $40 \mu \mathrm{g}$ PS-1145 samples.

The production of a porous PLGA scaffold by TIPS showed superior biodegradation compared to the previously used solid solvent-cast PDLLA. By 3 weeks the interior space contained trabecular bone and a pseudo-marrow cavity filled with cellular material with little residual polymer; previous work with solvent-cast PDLLA showed abundant residual polymer present at both the 3 week and 8 week time points[33]. This improved degradation was present in the rhBMP-2 alone group indicating this was due to the porous scaffold rather than the presence of PS-1145. The presence of woven bone with a high osteoclast number was not previously seen in the PDLLA solvent cast scaffolds and was most commonly observed in the local PS- 1145 treated group. It is not clear why the osteoclast number in these regions was so high (versus being reduced in the rest of the interior) or whether an analogous region was present at other time points in the rhBMP-2 alone group. It was surmised that the woven bone morphology observed at the experimental endpoint may be influenced by the presence of residual polymer scaffold.

This study is the first to examine synergy between rhBMP-2 and PS-1145 for bone tissue engineering. However, there are several reports of combining rhBMPs and bisphosphonates to coordinately modulate the anabolic and catabolic bone. In a rat critical defect model, treatment with Zoledronic Acid and rhBMP-7 (OP-1) was found to be superior to rhBMP-7 alone [32]. In a rat ectopic bone model, minodronate and rhBMP-2 delivered via a $\beta$-tricalcium phosphate scaffold resulted in increased net bone formation and mechanical strength compared to the delivery of a lone anabolic rhBMP-2 [51]. However, there remain concerns with bisphosphonate use in orthopedics in general [52], but also specifically due to their potential anti-angiogenic effects [53] and potential to delay hard callus remodeling [54]. 
Citation: Carr D, Yu NYC, Fitzpatrick J, Peacock L, Mikulec K, et al. (2011) Synergy between rhBMP-2 and IKK-Inhibitor PS-1145 Delivered via a Porous Biodegradable Polymer Implant. J Tissue Sci Eng S1:003. doi:10.4172/2157-7552.S1-003
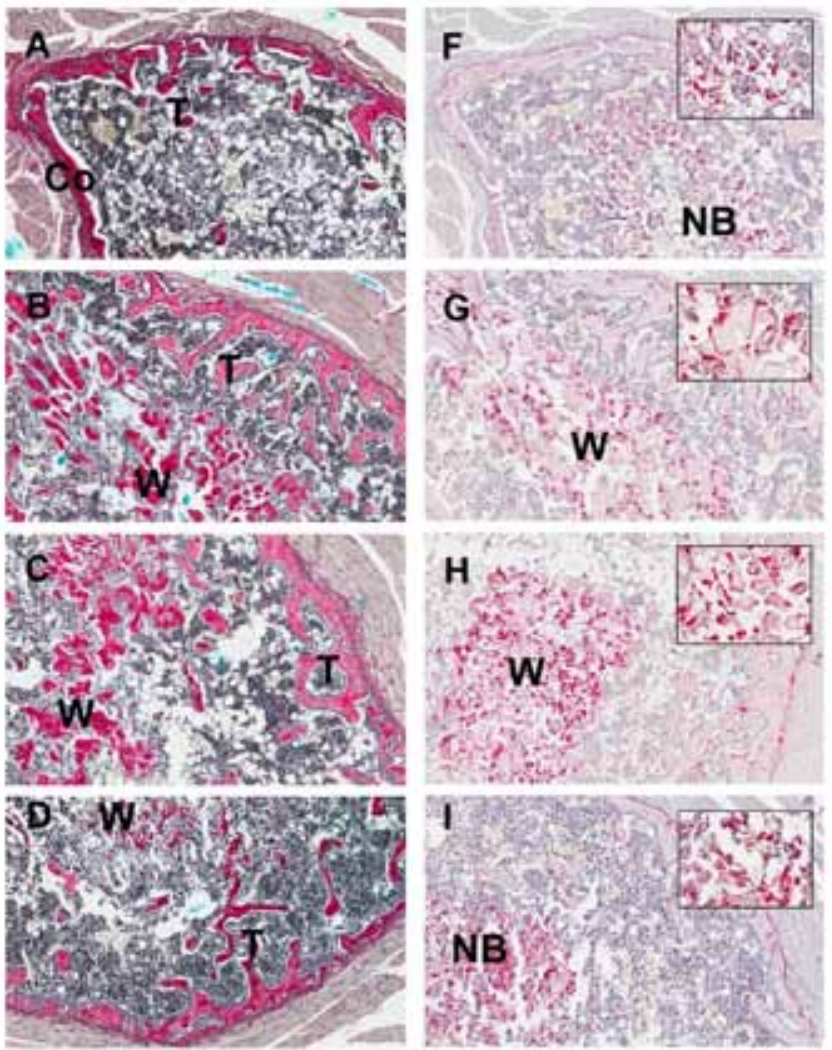

Figure 3: $A-E: A B / P R$ stained sections from groups 1-4 showing types of bone formation. F-I:TRAP stained sections from groups 1-4 showing large TRAP+ cell populations, with inset high power trabecular bone regions. Co, cortical bone; $T$, trabecular bone; W, woven bone; NB, no bone.

While the anti-resorptive effects on callus remodeling may be advantageous to prevent refracture or loss of fixation in the short term, prolonged ZA exposure may prevent the conversion of woven to lamellar bone even in appropriate loading conditions. In contrast, IKK inhibitors are not expected to be retained at bone surfaces. Transient exposure could delay bone remodeling to maximize bone in an unloaded environment, but allow for timely remodeling. In conclusion, IKK-inhibitors represent a new and exciting class of anti-catabolic agents for orthopaedic and bone tissue engineering applications.

\section{Acknowledgments}

Nicole Y. C. Yu was supported by the Australian National Health \& Medical Research Council (NHMRC) Biomedical Postgraduate Research Scholarship. Dr Schindeler receives salary support from NHMRC Project Grant APP1003478. This work was supported by NHMRC Project Grant APP1020987.

\section{References}

1. Porter JR, Ruckh TT, Popat KC (2009) Bone tissue engineering: a review in bone biomimetics and drug delivery strategies. Biotechnol Prog 25: 1539-1560.

2. Dell PC, Burchardt H, Glowczewskie FP Jr (1985) A roentgenographic, biomechanical, and histological evaluation of vascularized and nonvascularized segmental fibular canine autografts. J Bone Joint Surg Am 67: 105-112.

3. Giannoudis PV (2009) Fracture healing and bone regeneration: autologous bone grafting or BMPs? Injury 40: 1243-1244.

4. Hui JH, Ouyang HW, Hutmacher DW, Goh JC, Lee FH (2005) Mesenchymal stem cells in musculoskeletal tissue engineering: a review of recent advances in National University of Singapore. Ann Acad Med Singapore 34: 206-212.
5. Mazock JB, Schow SR, Triplett RG (2003) Posterior iliac crest bone harvest: review of technique, complications, and use of an epidural catheter for postoperative pain control. J Oral Maxillofac Surg 61: 1497-1503.

6. Salgado AJ, Coutinho OP, Reis RL (2004) Bone tissue engineering: state of the art and future trends. Macromol Biosci 4: 743-765.

7. Yoshikawa H, Myoui A (2005) Bone tissue engineering with porous hydroxyapatite ceramics. J Artif Organs 8: 131-136.

8. Yu NY, Schindeler A, Little DG, Ruys AJ (2010) Biodegradable poly(alphahydroxy acid) polymer scaffolds for bone tissue engineering. J Biomed Mater Res B Appl Biomater 93: 285-295.

9. Aronson J (1997) Limb-lengthening, skeletal reconstruction, and bone transport with the llizarov method. J Bone Joint Surg Am 79: 1243-1258.

10. de Pablos J, Barrios C, Alfaro C, Cañadell J (1994) Large experimental segmental bone defects treated by bone transportation with monolateral external distractors. Clin Orthop Relat Res 298: 259-265

11. Masquelet AC, Begue $T$ (2010) The concept of induced membrane for reconstruction of long bone defects. Orthop Clin North Am 41: 27-37.

12. Giannoudis PV, Einhorn TA, Marsh D (2007) Fracture healing: The diamond concept. Injury 38: S3-S6.

13. Liu R, Birke O, Morse A, Peacock L, Mikulec K, et al. (2011) Myogenic progenitors contribute to open but not closed fracture repair. BMC Musculoskelet Disord 12: 288.

14. Liu X, Ma PX (2004) Polymeric scaffolds for bone tissue engineering. Ann Biomed Eng 32: 477-486.

15. Zhang R, Ma PX (1999) Poly(alpha-hydroxyl acids)/hydroxyapatite porous composites for bone-tissue engineering. I. Preparation and morphology. J Biomed Mater Res 44: 446-455.

16. Lee JW, Gardella JA Jr (2002) Surface perspectives in the biomedica applications of poly(alpha-hydroxy acid)s and their associated copolymers. Anal Bioanal Chem 373: 526-537.

17. Zhang $P$, Hong $Z$, Yu T, Chen $X$, Jing $X$ (2009) In vivo mineralization and osteogenesis of nanocomposite scaffold of poly(lactide-co-glycolide) and hydroxyapatite surface-grafted with poly(L-lactide). Biomaterials 30: 58-70.

18. Whang K, Tsai DC, Nam EK, Aitken M, Sprague SM, et al. (1998) Ectopic bone formation via rhBMP-2 delivery from porous bioabsorbable polymer scaffolds. $J$ Biomed Mater Res 42: 491-499.

19. Francis L, Venugopal J, Prabhakaran MP, Thavasi V, Marsano E, et al. (2010) Simultaneous electrospin-electrosprayed biocomposite nanofibrous scaffolds for bone tissue regeneration. Acta Biomater 6: 4100-4109.

20. Bruder SP, Fox BS (1999) Tissue engineering of bone. Cell based strategies. Clin Orthop Relat Res : S68-83.

21. Sudarmadji N, Tan JY, Leong KF, Chua CK, Loh YT (2011) Investigation of the mechanical properties and porosity relationships in selective laser-sintered polyhedral for functionally graded scaffolds. Acta Biomater 7: 530-537.

22. Ma PX, Zhang R, Xiao G, Franceschi R (2001) Engineering new bone tissue in vitro on highly porous poly(alpha-hydroxyl acids)/hydroxyapatite composite scaffolds. J Biomed Mater Res 54: 284-293.

23. Giannoudis PV, Dinopoulos HT (2010) BMPs: Options, indications, and effectiveness. J Orthop Trauma 1: S9-16.

24. Gerstenfeld LC, Cullinane DM, Barnes GL, Graves DT, Einhorn TA (2003) Fracture healing as a post-natal developmental process: molecular, spatial, and temporal aspects of its regulation. J Cell Biochem 88: 873-884.

25. Ristiniemi J, Flinkkilä T, Hyvönen P, Lakovaara M, Pakarinen H, et al. (2007) RhBMP-7 accelerates the healing in distal tibial fractures treated by external fixation. J Bone Joint Surg Br 89: 265-272.

26. Govender S, Csimma C, Genant HK, Valentin-Opran A, Amit Y, et al. (2002) Recombinant human bone morphogenetic protein-2 for treatment of open tibial fractures: a prospective, controlled, randomized study of four hundred and fifty patients. J Bone Joint Surg Am 84: 2123-2134.

27. Friedlaender GE, Perry CR, Cole JD, Cook SD, Cierny G, et al. (2001) Osteogenic protein-1 (bone morphogenetic protein-7) in the treatment of tibial nonunions. J Bone Joint Surg Am 83: S151-158.

28. Wutzl A, Brozek W, Lernbass I, Rauner M, Hofbauer G, et al. (2006) Bone 
Citation: Carr D, Yu NYC, Fitzpatrick J, Peacock L, Mikulec K, et al. (2011) Synergy between rhBMP-2 and IKK-Inhibitor PS-1145 Delivered via a Porous Biodegradable Polymer Implant. J Tissue Sci Eng S1:003. doi:10.4172/2157-7552.S1-003

morphogenetic proteins 5 and 6 stimulate osteoclast generation. J Biomed Mater Res A 77: 75-83

29. Seeherman HJ, Li XJ, Bouxsein ML, Wozney JM (2010) rhBMP-2 induces transient bone resorption followed by bone formation in a nonhuman primate core-defect model. J Bone Joint Surg Am 92: 411-426.

30. Chamay A, Tschantz $P$ (1972) Mechanical influences in bone remodeling Experimental research on Wolff's law. J Biomech 5: 173-180.

31. Amanat N, Brown R, Bilston LE, Little DG (2005) A single systemic dose of pamidronate improves bone mineral content and accelerates restoration of strength in a rat model of fracture repair. J Orthop Res 23: 1029-1034.

32. Little DG, McDonald M, Bransford R, Godfrey CB, Amanat N (2005) Manipulation of the anabolic and catabolic responses with OP-1 and zoledronic acid in a rat critical defect model. J Bone Miner Res 20: 2044-2052.

33. Yu NY, Schindeler A, Peacock L, Mikulec K, Baldock PA, et al. (2010) In vivo local co-delivery of recombinant human bone morphogenetic protein-7 and pamidronate via poly-D, L-lactic acid. Eur Cell Mater 20: 431-441.

34. Choi JY, Kim HJ, Lee YC, Cho BO, Seong HS, et al. (2007) Inhibition of bone healing by pamidronate in calvarial bony defects. Oral Surg Oral Med Oral Pathol Oral Radiol Endod 103: 321-328.

35. Jimi E, Ghosh S (2005) Role of nuclear factor-kappaB in the immune system and bone. Immunol Rev 208: 80-87.

36. Chang J, Wang Z, Tang E, Fan Z, McCauley L, et al. (2009) Inhibition of osteoblastic bone formation by nuclear factor-kappaB. Nat Med 15: 682-689.

37. Yemelyanov A, Gasparian A, Lindholm P, Dang L, Pierce JW, et al. (2006) Effects of IKK inhibitor PS1145 on NF-kappaB function, proliferation, apoptosis and invasion activity in prostate carcinoma cells. Oncogene 25: 387-398.

38. Idris Al, Krishnan M, Simic P, Landao-Bassonga E, Mollat $P$, et al. (2010) Smal molecule inhibitors of I kappaB kinase signaling inhibit osteoclast formation in vitro and prevent ovariectomy-induced bone loss in vivo. FASEB J 24: 45454555

39. Idris $\mathrm{Al}$, Libouban $\mathrm{H}$, Nyangoga $\mathrm{H}$, Landao-Bassonga $\mathrm{E}$, Chappard $\mathrm{D}$, et al. (2009) Pharmacologic inhibitors of IkappaB kinase suppress growth and migration of mammary carcinosarcoma cells in vitro and prevent osteolytic bone metastasis in vivo. Mol Cancer Ther 8: 2339-2347.

40. Ruocco MG, Maeda S, Park JM, Lawrence T, Hsu LC, et al. (2005) I\{kappa B kinase $(\mathrm{IKK})\{$ beta\}, but not IKK\{alpha\}, is a critical mediator of osteoclast survival and is required for inflammation-induced bone loss. J Exp Med 201: 1677-1687.

41. Dai S, Hirayama T, Abbas S, Abu-Amer Y (2004) The IkappaB kinase (IKK) inhibitor, NEMO-binding domain peptide, blocks osteoclastogenesis and bone erosion in inflammatory arthritis. J Biol Chem 279: 37219-22.
42. von Metzler I, Krebbel H, Hecht M, Manz RA, Fleissner C, et al. (2007) Bortezomib inhibits human osteoclastogenesis. Leukemia 21: 2025-2034.

43. Cao Y, Mitchell G, Messina A, Price L, Thompson E, et al. (2006) The influence of architecture on degradation and tissue ingrowth into three-dimensional poly(lactic-co-glycolic acid) scaffolds in vitro and in vivo. Biomaterials 27: 2854 2864

44. Jack KS, Velayudhan S, Luckman P, Trau M, Grøndahl L, et al. (2009) The fabrication and characterization of biodegradable HA/PHBV nanoparticlepolymer composite scaffolds. Acta Biomaterialia 5: 2657-2667.

45. Schindeler A, Ramachandran M, Godfrey C, Morse A, McDonald M, et al (2008) Modeling bone morphogenetic protein and bisphosphonate combination therapy in wild-type and Nf1 haploinsufficient mice. J Orthop Res 26: 65-74.

46. Itoh K, Udagawa N, Katagiri T, lemura S, Ueno N, et al. (2001) Bone morphogenetic protein 2 stimulates osteoclast differentiation and survival supported by receptor activator of nuclear factor-kappaB ligand. Endocrinology 142: $3656-3662$

47. Jensen ED, Pham L, Billington CJ Jr, Espe K, Carlson AE, et al. (2010) Bone morphogenic protein 2 directly enhances differentiation of murine osteoclas precursors. J Cell Biochem 109: 672-82.

48. Clohisy JC, Yamanaka Y, Faccio R, Abu-Amer Y (2006) Inhibition of IKK activation, through sequestering NEMO, blocks PMMA-induced osteoclastogenesis and calvarial inflammatory osteolysis. J Orthop Res 24 1358-65.

49. Yip KH, Zheng MH, Feng HT, Steer JH, Joyce DA, et al. (2004) Sesquiterpene lactone parthenolide blocks lipopolysaccharide-induced osteolysis through the suppression of NF-kappaB activity. J Bone Miner Res 19: 1905-16.

50. Halleen JM, Tiitinen SL, Ylipahkala H, Fagerlund KM, Väänänen HK (2006) Tartrate-resistant acid phosphatase $5 b$ (TRACP $5 b$ ) as a marker of bone resorption. Clin Lab 52: 499-509.

51. Chen WJ, Jingushi S, Hirata G, Matsumoto $Y$, Iwamoto $Y(2004)$ Intramuscula bone induction by the simultaneous administration of recombinant human bone morphogenetic protein 2 and bisphosphonate for autobone graft. Tissue Eng 10: $1652-61$

52. Narongroeknawin $P$, Danila Ml, Humphreys LG Jr, Barasch A, Curtis JR (2010) Bisphosphonate-associated osteonecrosis of the jaw, with healing after teriparatide: a review of the literature and a case report. Spec Care Dentist 30 $77-82$

53. Walter C, Pabst A, Ziebart T, Klein M, Al-Nawas B (2011) Bisphosphonate affect migration ability and cell viability of HUVEC, fibroblasts and osteoblasts in vitro. Oral Dis 17: 194-199.

54. McDonald MM, Dulai S, Godfrey C, Amanat N, Sztynda T, et al. (2008) Bolus or weekly zoledronic acid administration does not delay endochondral fracture repair but weekly dosing enhances delays in hard callus remodeling. Bone 43 : 653-662.
This article was originally published in a special issue, Bone Tissue Engineering handled by Editor(s). Dr. Aaron Schindeler, Kids Research Institute, Australia 